\title{
nature
}

\section{Illuminating the black box}

\author{
Note to biologists: submissions to Nature should contain complete \\ descriptions of materials and reagents used.
}

Tros his journal aims to publish papers that are not only interesting and thought-provoking, but reproducible and useful. In order to do this, novel materials and reagents need to be carefully described and readily available to interested scientists.

That might seem obvious. But despite the efforts of our editors and referees, papers in the biological sciences are still being submitted - and occasionally published - that do not adequately describe the reagents used. Unless efforts are redoubled to eliminate this practice, we could see an era of 'black box' biology, in which outside researchers cannot work out what was done in an experiment.

Some of these 'rogue reagents' are the offspring of well-established technologies that have fallen under commercial control. A frequent example involves antibodies, reagents used throughout biology. The standard for publication should be that the authors can show an antibody's specificity and utility. But this information is sometimes missing from published papers, and readers must rely on a description from a company with a stake in the antibody in question.

But the problem is most acute in the case of new technologies, which sometimes experience a period of rapid development during which the rules just don't seem to apply. The desire to exploit the latest innovation can outstrip a field's ability to impose a common standard for description and documentation.

\section{Strictly business}

An example is RNA interference ( $\mathrm{RNAi}$ ), which allows researchers to manipulate gene expression. The critical reagents in RNAi studies are short interfering RNAs, the sequences of which determine which genes are affected. The approach has unleashed a frenzy of papers describing RNAi strategies that illuminate questions in biological systems and could have commercial application.

Manuscripts submitted from the RNAi field show a pattern typical of such new technologies. As the technology becomes commercialized, transparency slips. Nature journals have reviewed too many submissions with reagents incompletely described, or where material-transfer agreements limited availability.

Authors face conflicting pressures. Journals set publication standards for openness and availability, but many researchers have personal business interests, universities want to turn research into patent revenue, and biotechnology companies want to keep as much as possible under wraps.

In one case, an author published a paper without sequences of the RNAi reagents used. Editors and referees missed this. A year or so after publication, the author could not meet requests for his reagents, or provide the sequences for his short interfering RNAs. When the journal editors learned of this they asked for the sequences to be added to the paper. But the author said that patent issues and a business deal prevented him from doing so. The author's lab could not produce the short interfering RNAs, and a company that he had established didn't want the author to reveal the sequences, as this would jeopardize its raison d'être. This kind of stalemate matters, because it prevents the replication of experiments and inhibits the selection of appropriate controls in subsequent work.

Some authors claim replication is possible without full sequence information or the details of novel compounds. They say that the materials in question are for sale, enabling anyone to duplicate the paper. This misses the point. Scientific progress revolves around producing data that can drive the next stage of investigation. Even if consistent results can be achieved with a black-box reagent, knowing the composition and sequence can be the difference between making an observation and drawing insightful conclusions about it.

\section{False premise}

Another specious claim is that putting the structures of chemical compounds or sequences for short interfering RNAs in a manuscript is unnecessary, because if appropriate controls are described other investigators will know how to control their experiments. This is a false premise: the controls for an experiment designed to test one hypothesis are likely to be different from those needed to tackle another problem. To design appropriate controls one must know
"The desire to exploit the latest innovation can outstrip a field's ability to impose a common standard for description and documentation." what to control for - information that comes only from structures or sequences.

A troubling situation can also arise when a paper is published and the reagent provided, but a project ending or a person moving ends this provision. Journals have to decide whether to push for continued provision, and, if so, how. Is there a sensible statute of limitations on an author's commitment to provide? Input from the community would help publishers make a sensible policy; comments can be sent to authors@nature.com.

In the meantime, researchers must, when they are arranging the commercialization of their work, bear in mind the implications that these deals may have on their freedom to publish to the standards that the community is entitled to expect.

Nature will continue to require that, at acceptance, materials should be fully described in the paper or in the online Supplementary Information. If limited disclosure is noted between acceptance and publication, refusal to provide information may forfeit any offer to publish. Non-disclosure or inaccessibility brought to the editor's attention after publication will be investigated and actions taken, including, if necessary, public rebukes posted online and appended to the archived paper. Authors are expected to make reasonable arrangements, described accurately in the published work, by which readers can obtain the reagents described in their papers. 